\title{
Roberts of Lovedale and Eclipsing Binary Stars
}

\author{
Thomas R. Williams
}

3621 Wakeforest, Houston, TX 77098-5509, U.S.A.

Alexander William Roberts, a great humanitarian and teacher in South Africa, was also a luminary in the astronomical world. This paper discusses his work on variable stars and touches briefly on his career in other areas. Born and educated in Scotland, Roberts migrated to South Africa in 1883, at age 25, to teach at the Native College at Lovedale. He studied mathematical astronomy as a recreational pursuit, but became an active observer in 1889. After two years of general observing, be began a systematic survey using binoculars and an old one-inch theodolite. He carefully plotted all visible stars in selected areas and ranked them in order of their apparent brightness, doing so repeatedly on six evenings for each of the selected areas. His composite sketch became a reference chart as he searched for changes in these fields. With this technique, Roberts discovered more than 20 variable stars.

Early in his observing career, Roberts emphasized the importance of technique in visual photometry, a topic which he pursued in several papers on position-angle and field-orientation effects. In practice he removed the effect of the relative field position of the variable star and the comparison star by using one normal eyepiece and another eyepiece that reversed the field. He then average the two results on each observation. Eventually, he averaged four to six estimates made from various position angles with a special prismatic telescope designed for precise visual photometry. His analysis of light curves, developed from these careful observations, formed the basis for his most significant contributions in eclipsing binary astronomy.

Roberts' contributions to eclipsing binary astronomy are illustrated in the controversy on V Puppis. This star's variability, first suspected by the English amateur astronomer Arthur Stanley Williams in 1886, was confirmed by E.C. Pickering in 1895 with a period of 3.445 days based on spectroscopic observations. However, Roberts published his period of 1.4544757 days in the April 1901 Astronomical Journal. Roberts apparently came under some pressure from other astronomers to explain the period discrepancy since the spectroscopic period was generally accepted as the final authority. However, he resolutely stood his ground, stating "I have spoken my last word on the subject". The question remained unresolved until 1905 when Pickering stated in a letter to Roberts that "our observations of the spectrum of V Puppis agree with the period you found for that star." The Third General Catalogue of Variable Stars (1970) gives 1.4544877 days as the period for V Puppis, in good agreement with Roberts' original value. The resolution of the V Puppis period 
was important in removing a cloud over other of Roberts' conclusions based on the same observational data, especially his 1901 Astrophysical Journal observation that the V Puppis stars were nearly in contact and were severely distorted into ellipsoidal shapes by gravitational tidal effects. His conclusion provided crucial observational proof of theories which had been proposed separately by Poincaré and Darwin on tidal distortions of the shapes of stars and other astronomical bodies.

Roberts made many other contributions regarding the shapes and orbits of binary stars. In an 1899 Astrophysical Journal article, he calculated the upper limits of the density of the individual stars that are components of four southern eclipsing binaries in which the stars are very extended and tenuous objects. He showed that a circular orbit establishes an upper limit on the average density of the stars. His upper bound densities are similar to those proposed by Henry Norris Russell in the same Astrophysical Journal. The value of Roberts' work on binary stars has been acknowledged by his contemporaries as well as modern binary-star astronomers including W.W. Campbell, Zdenek Kopal and V.P. Tsesevich.

While gaining recognition as an astronomer, Roberts' reputation was growing as a teacher and humanitarian. In his main profession as a teacher at Lovedale College for Natives, he was personally responsible for training over 4000 native teachers. In 1920, Jan Smuts, Prime Minister and Minister for Native Affairs, chose Roberts to represent the natives as a white Senator in the all-white Parliament, and appointed him to a three-man Native Commission and several special investigative commissions. Poor health and political considerations forced Roberts to retire from these positions in 1935. Roberts was recognized as the foremost authority on native questions in South Africa, and would deserve a secure place in the history of South Africa based solely on his humanitarian efforts. Thus his significant contributions to astronomy are all the more interesting. In the words of Jan Smuts: "After Roberts let no amateur despair, and let each cultivate his scientific hobby to the utmost limit of his powers and opportunities. Science ... owes much to the amateur. Roberts was truly a prince among our scientific amateurs."

\section{Reference}

Williams, T.R.: Alexander Roberts: distinguished South African amateur astronomer and humanitarian, Abstract 01.07, Bull. Am. astron. Soc., 17 (4), 1985

An full bibliography regarding Roberts may be obtained from the author. 